\title{
SUBCONJUNCTIVAL BUPIVACAINE VERSUS TOPICAL AMETHOCAINE IN STRABISMUS SURGERY
}

\author{
N. E. HABIB, H. T. EL-KASABY, A. M. MARCZAK and J. HSUAN \\ Wolverhampton
}

\begin{abstract}
SUMMARY
A prospective study was performed to evaluate and compare the effect of subconjunctival bupivacaine and topical amethocaine on pain relief after strabismus surgery. A total of $\mathbf{4 0}$ children scheduled for elective operation were randomly allocated to receive either subconjunctival bupivacaine or topical amethocaine at the end of surgery. Post-operative pain was evaluated using a fourpoint assessment score. We show that both techniques provide good post-operative pain relief. We advocate that either could be used routinely in strabismus surgery although topical amethocaine is easier to administer.
\end{abstract}

The provision of optimal post-operative analgesia for children undergoing strabismus surgery is an important objective; post-operative pain is unpleasant and may have adverse physiological and psychological effects. Good pain relief will speed recovery, reduce post-operative emotional disturbance and may facilitate early discharge. ${ }^{1}$ Pain following many paediatric procedures can be effectively treated by local or regional analgesic techniques, ${ }^{2}$ and often no other medication will be required. This avoids the side effects of narcotics, allowing the child to return rapidly to full activity after surgery.

Recently, we reported ${ }^{3}$ that a long-acting anaesthetic, such as bupivacaine $0.5 \%$ injected subconjunctivally at the conclusion of strabismus surgery, significantly reduces post-operative pain. In this paper we continue our investigation of the optimum perioperative analgesia after strabismus surgery in children. As topical amethocaine is used routinely to provide conjunctival anaesthesia in ophthalmic examination and surgery, we aimed to compare the effect of subconjunctival bupivacaine and topical amethocaine on post-operative pain relief in strabismus surgery in children.

\section{PATIENTS AND METHODS}

Approval for this study was granted by the Local District

From: Wolverhampton and Midland Counties Eye Infirmary, Compton Road, Wolverhampton WV3 9QR, UK.

Correspondence to: Mr. H. El-Kasaby, FRCS, FRCOphth, Birmingham and Midland Eye Hospital, Church Street, Birmingham B3 2NS, UK
Ethics Committee and informed consent was obtained from the parents of all the children. A total of 40 children undergoing elective two-muscle horizontal strabismus correction surgery were included in the study. There were 22 girls and 18 boys with a mean age of 4.7 years (range 2-8 years). Age and sex distribution in the two groups did not differ significantly. Patients presenting for repeat surgery were excluded.

Patients were randomly allocated to two groups of 20, one of which received subconjunctival bupivacaine at the end of surgery and the other topical amethocaine. Randomisation instructions were placed in sealed envelopes at the beginning of the trial, which were then mixed and numbered and placed in the operating theatre. A control group in which patients received a subconjunctival injection of $0.25 \mathrm{ml}$ normal saline $(\mathrm{NaCl} \mathrm{BP} 0.9 \%$ ) over each operated muscle was presented in our previous report; ${ }^{3}$ this was not repeated in the current study so as not to subject our patients to unnecessary pain.

All patients were in group 1 according to the American Society of Anesthesiologists classification of physical status, ${ }^{4}$ indicating good general health. Both groups received Emla cream (Astra) to the proposed venepuncture site. Induction was either intravenous or inhalational according to the anaesthetist's preference. Anaesthesia was maintained with the patient spontaneously breathing in oxygen, nitrous oxide and volatile agent. During the procedure the patients were monitored using electrocardiography, pulse oximetry and non-invasive blood pressure monitoring. Short-acting intravenous analgesics which are effective for less than 30 minutes were used if indicated. Post-operative analgesia was prescribed on an 'as required' basis and consisted of paracetamol suspension orally (age $2-5$ years, $120-250 \mathrm{mg}$; 6-10 years, 250$500 \mathrm{mg}$ ) or intramuscular pethidine $1 \mathrm{mg} / \mathrm{kg}$.

Surgery was performed by four experienced surgeons (Consultant/Senior Registrar). The surgical technique was uniform in all operations including identical conjunctival approach and suture material. At the conclusion of surgery, the first group received a subconjunctival injection of 


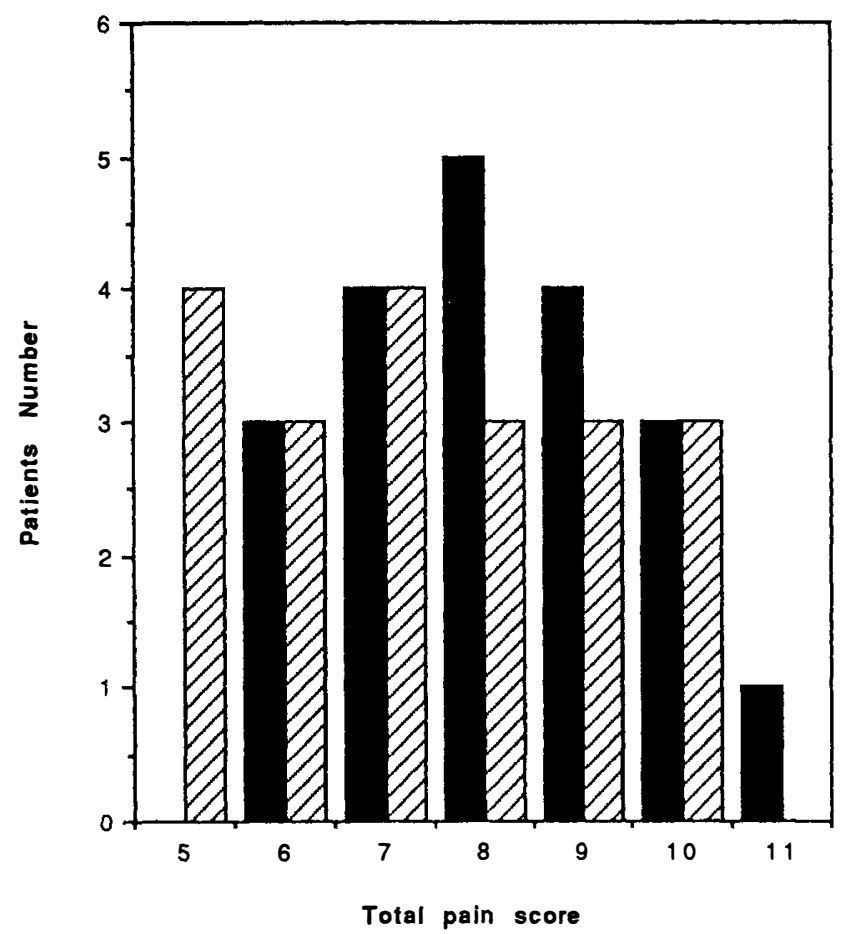

Fig. 1. Total post-operative pain assessment scores. Black columns, amethocaine; hatched columns, bupivacaine.

$0.25 \mathrm{ml}$ bupivacaine hydrochloride BP $0.5 \%$ over each operated muscle. The second group received two drops of $1 \%$ amethocaine hydrochloride into the eye being corrected, prior to and at the conclusion of surgery. No other topical medications were applied in either group and none of the eyes were padded.

The patients were always assessed for pain by the same senior ward nurse at 30 minutes, 1,2 and 4 hours after surgery. Assessment was made on a four-point scale: 1 , sleeping; 2 , awake and quiet; 3 , agitated; 4 , crying. The assessor nurse was masked to the randomisation. There were four scores for each patient, one at each of the four different time intervals. These were then summed to produce a total pain score. The higher the total score, the more uncomfortable the patient would have been. Statistical analysis of the assessment scores was undertaken using the two-sample $t$-test. At the outset of the study a mean difference of 2.0 between the total score in each group was considered as the target for a clinically significant difference. The trial was to stop if the observed difference was significantly less than 2.0 after 40 patients.

\section{RESULTS}

The mean of the total scores for the bupivacaine group as assessed by the nurse was 7.35 (range 5-10) and for the amethocaine group 8.15 (range 6-11) (Fig. 1). The mean difference was 0.80 (standard error 0.51 ). At this point in the study, statistical analysis of the total scores in the two groups showed no significant difference (two-sample $t$-test, $t=1.57,38$ d.f., $p=0.13$ ). Although subconjunctival bupivacaine consistently achieved better analgesia than amethocaine at the 30 minute, 1,2 and 4 hour inter-

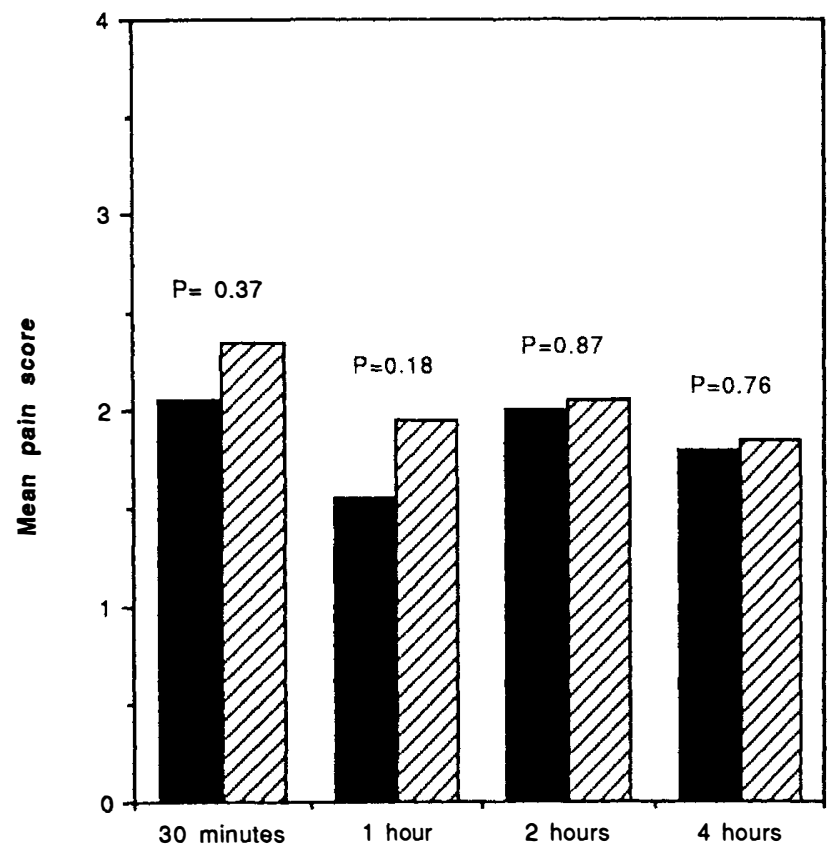

Fig. 2. Mean pain scores in the two groups as measured at different time intervals. Black columns, bupivacaine; hatched columns, amethocaine.

vals after surgery, this did not reach statistical significance (Fig. 2).

At the beginning of the study, it was proposed in the protocol that a mean difference of 2.0 was required to label one method more effective than the other. The observed difference in the total scores of the two groups was significantly less than the expected difference of $2.0(t=-2.35$, $p=0.01$ ).

There was no significant difference between the two groups in patients requiring oral paracetamol suspension for analgesia. No patient in either group required pethidine.

\section{DISCUSSION}

Local anaesthesia by wound infiltration is a well-established technique for post-operative pain relief. It is widely used at the conclusion of a multitude of surgical procedures in children including inguinal, umbilical, penile and perineal surgery. ${ }^{5}$ Subconjunctival bupivacaine administered at the conclusion of strabismus surgery over each operated muscle has already been shown to be effective in reducing post-operative pain and discomfort. Although special attention to the total drug dose is required, as it is very easy to approach toxic levels of local analgesics in children, the dose used following strabismus surgery is well below toxic levels.

Deep structures such as sclera and Tenon's fascia, as well as stretching of rectus muscles as in resection procedures, may contribute to post-operative discomfort. ${ }^{6}$ However, it is probably pain receptors in the conjunctiva that are the main afferent pathways of post-operative pain. Amethocaine is used extensively in ophthalmology as a 
topical anaesthetic in examination and surgery. Watson ${ }^{7}$ showed that topical amethocaine administered at the conclusion of strabismus surgery decreases the requirement for intramuscular opiates in the post-operative period. Amethocaine has two advantages over bupivacaine subconjunctivally: it is easier to administer and carries no potential risk for inadvertent subconjunctival haemorrhage.

Our study shows that bupivacaine $0.5 \%$ injected subconjunctivally at the conclusion of strabismus surgery and topical amethocaine administered at the beginning and conclusion of surgery are equally effective in significantly reducing post-operative pain. No systemic or local side effects were encountered in any of the patients.

Premedications and long-acting opiates were not allowed in this trial. This avoids sedation, nausea and vomiting and also makes pain assessment easier. As in our previous study, because of the many children younger than 4 years, assessment by an observer using the four-point assessment score was the only feasible method.

Subconjunctival bupivacaine or topical amethocaine are easily administered at the conclusion of strabismus surgery and provide good post-operative analgesia. We recommend that either may be used routinely, especially in day case surgery.
The authors wish to thank Mr. P. I. Murray for critically reading the manuscript, and Ms. H. S. Jones for her help with statistical analysis of the data.

Key words: Amethocaine, Analgesia, Bupivacaine, Post-operative pain, Strabismus, Surgery.

\section{REFERENCES}

1. Hadaway EG, Ingram RM, Traynar MJ. Day case surgery for strabismus in children. Trans Ophthalmol Soc UK 1977;97: $23-5$.

2. Fell D, Derrington MC, Tylor E, Wandless JG. Paediatric postoperative analgesia: a comparison between caudal block and wound infiltration of local anaesthetic. Anaesthesia 1988;43:107-10.

3. El-Kasaby HT, Habib NE, Marczak AM. Subconjunctival bupivacaine in strabismus surgery. Eye 1993;7:346-9.

4. American Society of Anesthesiologists. New classification of physical status. Anesthesiology 1963;24:111.

5. Hashemi K, Middleton MD. Subcutaneous bupivacaine for postoperative analgesia after herniorrhaphy. Ann R Coll Surg Engl 1983;65:38-9.

6. Burton H. Somatosensory sensation from the eye. In: Moses RA, editor. Adler's physiology of the eye: clinical applications. 7th ed. St. Louis: CV Mosby, 1981:74.

7. Watson DM. Topical amethocaine in strabismus surgery. Anaesthesia 1991;46:368-70. 